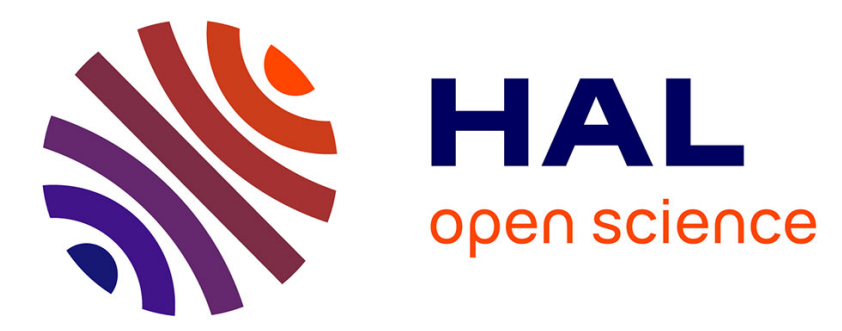

\title{
Adaptive Target Detection Techniques for OFDM-Based Passive Radar Exploiting Spatial Diversity
}

Gilles Chabriel, Jean Barrère

\section{To cite this version:}

Gilles Chabriel, Jean Barrère. Adaptive Target Detection Techniques for OFDM-Based Passive Radar Exploiting Spatial Diversity. IEEE Transactions on Signal Processing, 2017, 65 (22), pp.5873 - 5884. 10.1109/TSP.2017.2742980 . hal-01790695

\section{HAL Id: hal-01790695 \\ https://hal-amu.archives-ouvertes.fr/hal-01790695}

Submitted on 13 May 2018

HAL is a multi-disciplinary open access archive for the deposit and dissemination of scientific research documents, whether they are published or not. The documents may come from teaching and research institutions in France or abroad, or from public or private research centers.
L'archive ouverte pluridisciplinaire HAL, est destinée au dépôt et à la diffusion de documents scientifiques de niveau recherche, publiés ou non, émanant des établissements d'enseignement et de recherche français ou étrangers, des laboratoires publics ou privés. 


\section{Adaptive Target Detection Techniques for OFDM-Based Passive Radar Exploiting Spatial Diversity

\author{
Gilles Chabriel and Jean Barrère
}

\begin{abstract}
Recently, it has been studied the benefit of using a channel-based detector (CHAD) with a passive radar exploiting OFDM waveforms radiated e.g. by DVB-T transmitters of opportunity.

When multiple antennas are available on receive, we state that CHAD can be seen as space-time array-processing performing on a particular coherent frequency datacube. Building on this new interpretation, we propose an improved version of CHAD in the form of a fully dimensional space-time adaptive processing. Optimization of the signal-to-interference-plus-noise ratio is obtained combining a linearly constrained minimum variance (LCMV) space-time adaptive beamforming and a least squares (LS) spatial adaptive filtering.

Unlike classical STAP approaches, no training data are here used and only one space-time sample matrix inversion is required. The computational load is then highly reduced allowing a practical deployment. Moreover, since no beamscan in space is performed, the knowledge of array characteristics is not required and the performance shall not be impacted by any calibration errors. Finally, the full set of target signal returns being collected in a single range-Doppler surveillance map, the detection process is then simplified.
\end{abstract}

Results on experimental data show the interests of this new surveillance scheme: no need for a prior rejection of the dominant interference, systematic reduction of secondary lobes, discrimination of slow moving targets.

Index Terms-passive bistatic radar (PBR), passive covert radar (PCR), passive coherent location (PCL), digital video broadcasting-terrestrial (DVB-T), orthogonal frequency division multiplexing (OFDM), spatial diversity, cross-ambiguity function (CAF), matched filtering, mismatched filter, reciprocal filter, clutter, linearly constrained minimum variance (LCMV) beamforming, CAPON's receptor, array-processing, space-time adaptive processing (STAP), least squares (LS) filtering.

\section{INTRODUCTION}

$\mathbf{T}$ his work addresses the problem of moving targets detection from a passive radar that exploits OFDMbased sources of illumination through a Rician fading channel. For such systems, a main issue arises from the presence of powerful propagation paths, mainly zero-Doppler paths as the line-of-sight (LOS) path and secondary paths due to relief, vegetation, buildings, etc., that may prevent detection of the targets of interest; the detection peaks corresponding to the weakest target signal returns being often buried by the clutter floor induced by these dominant paths.

Gilles Chabriel and Jean Barrère are with Aix Marseille Université, CNRS, Université de Toulon, IM2NP UMR 7334, CS 60584 - 83041 Toulon Cedex 9, France (e-mail: gilles.chabriel@im2np.fr; jean.barrere@im2np.fr).
When the surveillance system involves multiple antennaelements (say $R$ ), space-time adaptive processing (STAP) theoretically offers a high-performing solution (see e.g. [1], [2] for airborne radars and [3] for ground radars). STAP performs from a $(R \times M \times K)$-datacube obtained by cutting out each -received signal $r$ into $M$ consecutive blocks (pulses) with $K$ samples each. In order to assure temporal coherence within the datacude, the different pulses are classically matched filtered using the corresponding block of the reference signal i.e. the transmitted signal, assumed to be known. The space-time adaptive processor aims to exploit spatial diversity and thus to reach signal-to-interference-plusnoise ratio (SINR) optimization. It consists to implement for each range cell under test (one of the $K(R \times M)$ )-slices of the datacube), a battery of space-time filters depending on Doppler shift and on at least one spatial parameter (a desired direction of arrival). For each Doppler shift and each direction, the optimal space-time adaptive filter is ideally built from the corresponding space-time steering vector and the inverse of the interference plus noise space-time covariance matrix. In the so-called sample matrix inversion (SMI) algorithm, this matrix is estimated averaging space-time snapshots (training data) taken in the vicinity of the under-test cell.

Beside a high theoretical performance, SMI raises major practical issues. It is well known that a very important computational complexity is reached since for each range bin, a full dimension space-time covariance matrix has to be estimated and inverted. Moreover, the amount of available homogeneous training data is not always sufficient with respect to the number of degrees-of-freedom $(\mathrm{DoF})$ brought by the space-time array. Hence, various DoF reduction strategies are used, conducting to suboptimal practical implementations and a dominant interference rejection prestage is often necessary before STAP. For ground radars, this dominant interference is mostly caused by zero-Doppler contribution (ZDC). Note that space-time approaches based on cross-ambiguity function [4], [5], [6], [7], [8] also recommend a ZDC rejection. For airborne radars, rejection of the Doppler shifted strongest contributions is also required with the risk that some targets of interest (often in practice the slowest moving targets) fall in the rejection band of the filter [9].

One can find e.g. in [10], [11], [12], [13], different methods for such a ZDC rejection. It can however be highlighted that these preprocessings are in general sensitive to antenna impairments such as mechanical vibrations or RF front-end impairments such as carrier frequency offset (CFO) or sample 
frequency offset (SFO). So, one cannot expect to reach in practice a full ZDC rejection.

A common point between all these approaches is the need to know the geometry of the (necessary calibrated) array so that beams in space can be scanned.

In this paper and as in [14] where a particular spatial extension of classical cross-ambiguity function (CAF) is proposed, we choose to address the problem of target detection in a blind way i.e. without any use of spatial (angular) parameterization. The classical spatial beamscan is then fully replaced by a minimization problem conducting directly to a unique (optimal) beam for each range-Doppler bin under test. Although the spatial information is lost or more accurately unused, such approaches have many advantages: target returns are always delivered in a single range-Doppler map, thereby simplifying the surveillance process by a human operator or an automatic tracker. Moreover, the performance can no longer be impacted by array calibration errors, as it can be with array-shape dependent methods. Finally and importantly, the computational load remains reasonably low when compared to those of classical STAP approaches.

For OFDM-based emissions, it has been proposed in [15], [16] to replace the battery of matched filters by "mismatched" filters (also called reciprocal filters). The idea is that reciprocal filter outputs being theoretically "signalindependent", presence of ghost targets (false alarms) caused by the DVB-T signal structure (cyclic prefix, transmission parameter signalling, pilot data, see [17]) is naturally eliminated. In [18], a new interpretation of such an approach was stated in term of channel-based detector (CHAD). Note that a similar interpretation can be found in [19] for the particular case of DAB signals. It was demonstrated that, thanks to the orthogonality between the subcarriers, CHAD has, in general, less clutter leakages than using matched filters. In particular, the "low-Dopplerized" paths induce a near zero clutter floor level; this level theoretically reaching zero for ZDC.

Here, we show that the multidimensional extension of CHAD can be seen as $R$ classical data-independent (not adaptive) beamformers in time performing from a coherent frequency datacube where the number $M$ of pulses corresponds to the number of OFDM symbols processed. From such an interpretation, we propose to replace the classical dataindependent beamformer by a linearly constrained minimum variance adaptive beamformer fully coupled with a least squares spatial adaptive filtering. We obtain a low complexity fully adaptive STAP free of any array spatial parameterization where the dominant interference prior rejection is no more required.

The paper is organized in the following way: Section II describes the spatial model on receive at baseband obtained under classical simplifying assumptions. The notion of Doppler channel is recalled and the theoretical expression of its frequency response is derived in the case of a DVB$\mathrm{T}$ emission. In the section III, two multidimensional rangeDoppler detection tools, namely space-time CAF and spacetime CHAD, are described. In Section IV we develop the proposed blind STAP (B-STAP) version of CHAD and we discuss its computational complexity. Examples of surveillance maps obtained with two experimental ground passive radars are presented in Section $\mathrm{V}$ where the performance of CAF (with and without ZDC rejection), of CHAD (with and without ZDC rejection) and finally of B-STAP CHAD are compared.

\section{PROBLEM MODELING \\ A. Far-field spatial model}

The schematic view in Fig. 1 illustrates a passive ground radar detection scenario where a transmitter of opportunity illuminates a scene composed of $I-1$ static or moving specular reflectors. A receive array-antenna composed of $R$ elements collects the contributions of the transmitted signal from $I$ propagation paths (the LOS path and $I-1$ secondary paths).

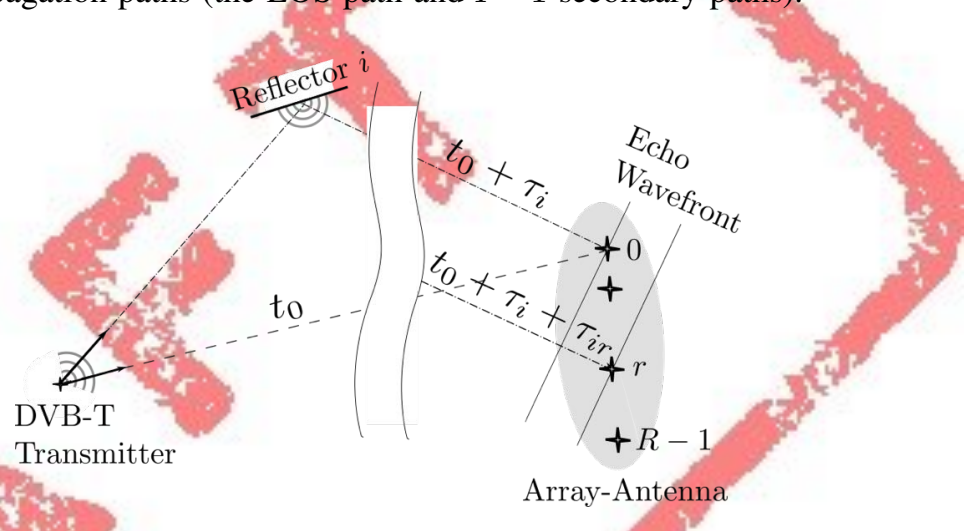

Fig. 1. Far-field modeling.

Let us introduce as a radar reference signal, the LOS complex baseband signal received on an element indexed by 0 , arbitrary chosen as a reference element. This signal denoted by $s(t)$, matches the complex baseband transmitted signal up to a propagation delay $t_{0}$ due to LOS distance.

Throughout this paper, we denote generically by $\mathcal{I}_{I}$, the set of indices $i=0, \ldots, I-1$, that is to say $\mathcal{I}_{I}=\{0,1, \ldots, I-1\}$. Assuming validity of some classical simplifying assumptions (see the appendix for more details), the complex baseband signal $y_{r}(t)$ received on any sensor $r \in \mathcal{I}_{R}$, is given by:

$$
y_{r}(t)=\sum_{i \in \mathcal{I}_{I}} \gamma_{i r} s\left(t-\tau_{i}\right) \mathrm{e}^{\jmath 2 \pi \mathrm{f}_{i} t}+\eta_{r}(t)
$$

where

- $i>0$ is the index of a reflector. It varies from 1 to $I-1$ and $i=0$ corresponds to the LOS path; $r$ is the index of an element of the receive array-antenna. It varies from 0 to $R-1$ ( $r=0$ being reserved for the reference element) ; a particular path is defined by the pair $(i, r)$;

- $\tau_{i}$ is the relative propagation delay such that $\tau_{i}+t_{0}$ corresponds to the propagation delay of the path $(i, 0)$ (i.e. from the transmitter to the reference sensor, via the $i^{\text {th }}$ reflector, see fig.1),

- $\mathrm{f}_{i}$ is the Doppler frequency shift due to the $i^{\text {th }}$ reflector bistatic speed,

- $\gamma_{i r}$ are spatial complex factors depending on relative propagation small delays, radar cross-sections (RCS) of 
the different reflectors and eventually on some impairments about the array-antenna such as e.g. the spatial

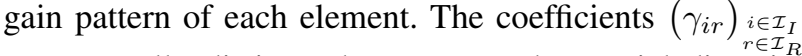
are generally distinct; they express the spatial diversity brought by the array-antenna.

- $\eta_{r}(t)$ is the contribution of the additive white Gaussian noise (AWGN).

\section{B. Notion of Doppler channel}

As introduced in [18], the previous model (1) can be rewritten as a sum of $F$ differently frequency shifted ("Dopplerized") contributions and one has using the convolution operator $*$ :

$$
y_{r}(t)=\sum_{j \in \mathcal{I}_{F}}\left\{h_{r}^{f_{j}} * s\right\}(t) \mathrm{e}^{\jmath 2 \pi f_{j} t}+\eta_{r}(t)
$$

where $f_{j}\left(j \in \mathcal{I}_{F}\right)$ is one of the distinct Doppler frequency shifts, and where $h_{r}^{f_{j}}(t)$ represents the impulse response of the linear filter that models the multipath between the source and the receiver $r$ at Doppler shift $f_{j}$. Such a filter characterizes the so-called Doppler channel associated with the element $r$ and corresponding to the Doppler frequency shift $f_{j}$. Introducing the index subset $\mathcal{J}_{f_{j}}$ of $\mathcal{I}_{I}$, corresponding to a given Doppler frequency shift $f_{j}$ such as

$$
\mathcal{J}_{f_{j}}=\left\{i \in \mathcal{I}_{I} / \mathfrak{f}_{i}=f_{j}\right\}
$$

we have from (1), $\forall j \in \mathcal{I}_{F}, \forall r \in \mathcal{I}_{R}$

$$
h_{r}^{f_{j}}(t)=\sum_{i \in \mathcal{J}_{f_{j}}} \gamma_{i r} \delta\left(t-\tau_{i}\right),
$$

where $\delta(t)$ represents the Dirac pseudo-function.

Presence of a propagation path $i$ with a Doppler shift $f_{j}$ and a bistatic delay $\tau_{i}$ is then betrayed by a pulse in the impulse response $h_{r}^{f_{j}}(t)$. Equivalently, if $\mathcal{F}\{$.$\} denotes the Fourier$ transform, the contribution of such a path ideally takes the form of a complex exponential in the corresponding frequency response $H_{r}^{f_{j}}(\nu) \triangleq \mathcal{F}\left\{h_{r}^{f_{j}}(t)\right\}$.

\section{The DVB-T particular case}

For DVB-T illuminators (see [20]), the digital reference signal is a sequence of so-called CP-OFDM symbols $s_{m}[n]$, $N$ samples each, such that

$$
\begin{array}{r}
s_{m}[n]=\frac{1}{K} \sum_{k \in \mathcal{I}_{K}} d_{m}^{k} \mathrm{e}^{\jmath 2 \pi \frac{k n}{K}} \\
n \in[-N+K, \ldots, 0, \ldots, K-1], m \in \mathcal{I}_{M},
\end{array}
$$

where $K$ is the number of orthogonal subcarriers with the frequency spacing $1 / T_{u}$. $\mathcal{I}_{K}$ defines the support of the useful part whose duration is $T_{u}$ and $[-N+K, \ldots,-1]$ defines the support of the cyclic prefix (CP) with a $T_{c p}$ duration. Finally, $d_{m}^{k}$ is the complex information datum that modulates the subcarrier $\frac{k}{T_{u}}$ during the emission of the symbol $m$. In practice, the useful part of the symbol $m$, i.e. $\left(s_{m}[n]\right)_{n \in \mathcal{I}_{K}}$, is built from the inverse discrete Fourier transform (IDFT) of the discrete sequence of data $\left(d_{m}^{k}\right)_{k \in \mathcal{I}_{K}}$, and the samples in the CP part $\left(s_{m}[n]\right)_{n \in[-N+K, \ldots,-1]}$, are obtained by a periodic extension of the useful part. In order to mitigate crosstalk issues between consecutive transmission channels, the lowest and highest frequency subcarriers have a zero amplitude and one can equivalently say that they are modulated by zero data. Hence, we denote by $\mathcal{E}_{K}$, the index subset of non-zero data: $\mathcal{E}_{K}=\left\{k \in \mathcal{I}_{K} / d_{m}^{k} \neq 0\right\}$.

As most of the authors on this topic, we assume here that all the required conditions for an error-free DVB-T demodulation are fulfilled [21]. As a consequence, the sampled version $y_{r}[n]$ (sample period: $T_{u} / K$ ) of the received signal is assumed to be synchronized to the begining of the useful part of the LOS dominant contribution and the whole dataset $\left(d_{m}^{k}\right)_{\substack{k \in \mathcal{E}_{K} \\ m \in \mathcal{I}_{M}}}$ is considered known. The digital reference signal $\left(s_{m}[n]\right)_{\substack{n \in \mathcal{I}_{K} \\ m \in \mathcal{I}_{M}}}^{\substack{m \\ \text { and }}}$ can then besynthesized by IDFT and periodic extension without any error.

Assuming that $\tau_{i}<T_{c p}, \forall i \in \mathcal{I}_{I}$, each sample ${ }^{\mathrm{a}}$ in the useful part of a received block $m$ on an element $r$ yields

$$
\begin{aligned}
y_{m r}[n]= & \sum_{j \in \mathcal{I}_{F}}\left[\frac{1}{K} \sum_{k \in \mathcal{E}_{K}} H_{r}^{f_{j}}[k] d_{m}^{k} \mathrm{e}^{2 \jmath \pi \frac{k n}{K}}\right] \mathrm{e}^{\jmath 2 \pi f_{j}\left(m T_{s}+n \frac{T u}{K}\right)} \\
& +\eta_{m r}[n], n \in \mathcal{I}_{K}
\end{aligned}
$$

with $T_{s} \triangleq T_{u}+T_{c p}$ and where

$H^{f_{j}}[k]=\sum T_{i r} \mathrm{e}^{-2}$

$$
H_{r}^{f_{j}}[k]=\sum_{i \in \mathcal{J}_{f_{j}}} \gamma_{i r} \mathrm{e}^{-2 \jmath \pi \frac{k \tau_{i}}{T_{u}}}, \quad k \in \mathcal{E}_{K}
$$

is the frequency response of the Doppler channel associated with the Doppler frequency shift $f_{i}$. The samples $\eta_{m r}[n]$ correpond to the additive noise contribution.

For sufficiently low Doppler shifts, it can be assumed that the phase rotation $\mathrm{e}^{j 2 \pi \mathrm{f}_{i} t}$ in (1) (or equivalently $\mathrm{e}^{32 \pi f_{j}\left(m T_{s}+n \frac{T u}{K}\right)}$ in (2)) remains constant within each CPOFDM block of duration $T_{s}$. Under this assumption, one can replace the phasor $\mathrm{e}^{j 2 \pi f_{j}\left(m T_{s}+n \frac{T u}{K}\right)}$ by $\mathrm{e}^{22 \pi f_{j} m T_{s}}$ in the expression (2). It comes

$$
\begin{gathered}
y_{m r}[n]=\sum_{j \in \mathcal{I}_{F}}\left[\frac{1}{K} \sum_{k \in \mathcal{E}_{K}} H_{m r}^{f_{j}}[k] d_{m}^{k} \mathrm{e}^{2 \jmath \pi \frac{k n}{K}}\right]+\eta_{m r}[n], \\
H_{m r}^{f_{j}}[k]=\sum_{i \in \mathcal{J}_{f_{j}}} \gamma_{i r} \mathrm{e}^{\jmath 2 \pi f_{j} m T_{s}} \mathrm{e}^{-2 \jmath \pi \frac{k \tau_{i}}{T_{u}}}, \quad k \in \mathcal{E}_{K}
\end{gathered}
$$

where

corresponds to the instantaneous frequency response of the $f_{j}$-Doppler channel varying from symbol to symbol.

\section{Multisensor RANGe-Doppler Detection tools}

In this section, we give the expressions of two rangeDoppler detection multidimensional (spatial) tools used in passive radar (CAF and CHAD) in which the knowledge of the array shape is not required.

\footnotetext{
${ }^{\text {a }}$ some of the samples for $T_{c p}<\tau_{i}<T_{c p}+T_{u}$
} 


\section{A. A "blind" cross-ambiguity function (CAF)}

Classical CAF is built from cross-correlations between the received signal $\left(y_{m r}[n]\right)_{\substack{n \in \mathcal{I}_{K} \\ m \in I_{M}}}$ on an element $r$ of the array and a bank of differently delayed $\left(\tau_{n^{\prime}}\right)$, differently frequency shifted $(f)$ versions of the power-normalized reference sequence $\left(s_{m}[n]\right)_{\substack{n \in \mathcal{I}_{K} \\ m \in \mathcal{I}_{M}}}$ :

$$
\mathcal{X}_{r}\left[n^{\prime}, f\right]=\frac{1}{K M} \sum_{\substack{n \in \mathcal{I}_{K} \\ m \in \mathcal{I}_{M}}} y_{m r}[n]\left(s_{m}\left[n-n^{\prime}\right]\right)^{*} \mathrm{e}^{-2 \jmath \pi f m T_{s}},
$$

with $\tau_{n^{\prime}}=n^{\prime} \frac{T_{u}}{K}$. Then, CAF expression is given by the square modulus of $\mathcal{X}_{r}\left[n^{\prime}, f\right]$ :

$$
\mathcal{A}_{r}\left[n^{\prime}, f\right]=\left|\mathcal{X}_{r}\left[n^{\prime}, f\right]\right|^{2} .
$$

A multidimensional (spatial), blind (not requiring any spatial model like steering vector), extension of $\mathrm{CAF}$ can be found in [14]. This approach consists in identifying the weight vector $\mathbf{w}=\left(w_{r}\right)_{r \in \mathcal{I}_{R}}$ of the least squares (LS) spatial filter minimizing the following mean square error:

$$
\varepsilon^{2}\left[n^{\prime}, f\right]=\frac{1}{M K} \sum_{\substack{n \in \mathcal{I}_{K} \\ m \in \mathcal{I}_{M}}}\left|\mathbf{w}^{H} \mathbf{y}_{m}[n]-s_{m}\left[n-n^{\prime}\right] \mathrm{e}^{2 \jmath \pi f m T_{s}}\right|^{2}
$$

where $\mathbf{y}_{m}[n]$ is the column vector of size $R$ gathering each $n^{\text {th }}$ sample of each $m^{\text {th }}$ pulse, received by the $R$ antennas: $\mathbf{y}_{m}[n]=\left(y_{m r}[n]\right)_{r \in \mathcal{I}_{R}}$.

Minimization of $\varepsilon^{2}$ yields:

$$
\mathbf{w}\left[n^{\prime}, f\right]=\boldsymbol{\Gamma}_{y}^{-1} \mathbf{X}\left[n^{\prime}, f\right],
$$

where the $R \times R$ matrix

$$
\boldsymbol{\Gamma}_{y} \triangleq \frac{1}{K M} \sum_{n \in \mathcal{I}_{K}} \sum_{m \in \mathcal{I}_{M}} \mathbf{y}_{m}[n] \hat{\mathbf{y}}_{m}^{H}[n]
$$

is an estimate of the spatial variance covariance matrix of the different recordings, and where $\mathbf{X}\left[n^{\prime}, f\right]=\left(\mathcal{X}_{r}\left[n^{\prime}, f\right]\right)_{r \in \mathcal{I}_{R}}$.

The blind space-time CAF is finally given by the energy of the spatially filtered observations

$$
\mathcal{A}_{\mathrm{ST}}\left[n^{\prime}, f\right]=\mathbf{X}^{H}\left[n^{\prime}, f\right] \mathbf{\Gamma}_{y}^{-1} \mathbf{X}\left[n^{\prime}, f\right] .
$$

It can be demonstrated that (5) gives the optimal solution, in the maximum likelihood ratio sense, when the problem would be to detect a single propagation path observed through multiple independent AWGN channels. Note that the outputs of this particular CAF remain in the range-Doppler plane unlike other approaches (see e.g. [4], [5], [6], [7], [8]) using CAF built on a spatial model where the angular parameters increase the output dimension.

\section{B. Doppler channel detector (CHAD)}

Let us denote by $Y_{m r}^{f}[k]$ the spectral representation of the so-called Doppler compensated sequence of samples $y_{m r}^{f}[n]=$ $y_{m r}[n] \mathrm{e}^{-2 \jmath \pi f m T_{s}}$ obtained using a $K$-points DFT:

$$
Y_{m r}^{f}[k]=\sum_{n \in \mathcal{I}_{K}} y_{m r}[n] \mathrm{e}^{-2 \jmath \pi f m T_{s}} \mathrm{e}^{-2 \jmath \pi \frac{k n}{K}} .
$$

From (2), an estimation of the instantaneous frequency response $\hat{H}_{m r}^{f}[k]$ of the $f$-Doppler channel is then given $\forall k \in$ $\mathcal{E}_{K}$, by

$$
\text { 1. } \hat{H}_{m r}^{f}[k]=\frac{Y_{m r}^{f}[k]}{d_{m}^{k}}
$$

and for the zero-amplitude subcarriers i.e. $\forall k \in \mathcal{I}_{K} \backslash \mathcal{E}_{K}$, by

$$
\hat{H}_{m r}^{f}[k]=0,
$$

It has been shown in [18] that when the number of symbols $M$ increases, the following summation

$$
\hat{H}_{r}^{f}[k] \triangleq \frac{1}{M} \sum_{m \in \mathcal{I}_{M}} \hat{H}_{m r}^{f}[k], k \in \mathcal{E}_{K},
$$

asymptotically converges to the frequency response $H_{r}^{f}[k]$ of the Doppler channel associated to the frequency shift $f$. Then, the square modulus of the corresponding impulse response $h_{r}^{f}\left[n^{\prime}\right]$ obtained by a $K$-points IDFT:

$$
\hat{h}_{r}^{f}\left[n^{\prime}\right]=\frac{1}{K} \sum_{k \in \mathcal{E}_{K}} \hat{H}_{r}^{f}[k] \mathrm{e}^{2 \jmath \pi \frac{k n^{\prime}}{K}}
$$

leads to the Doppler channel detector CHAD:

$$
\mathcal{C}_{r}\left[n^{\prime}, f\right]=\left|\hat{h}_{r}^{f}\left[n^{\prime}\right]\right|^{2},
$$

where the presence of a propagation path with a Doppler shift $f$ and a sample shift $n^{\prime}$ is ideally betrayed by a Dirac pulse.

As for CAF, we propose here a multidimensional (still blind) extension of CHAD searching the LS spatial filter $\mathbf{w}=\left(w_{r}\right)_{r \in \mathcal{I}_{R}}$ that minimizes the following quadratic error

$$
\varepsilon^{2}\left[n^{\prime}, f\right]=\sum_{k \in \mathcal{E}_{K}}\left|\mathbf{w}^{H} \mathbf{H}^{f}[k]-\mathrm{e}^{-2 \jmath \pi \frac{k n^{\prime}}{K}}\right|^{2},
$$

where $\mathbf{H}^{f}[k]$ is the column vector of size $R$ such that $\mathbf{H}^{f}[k]=\left(\hat{H}_{r}^{f}[k]\right)_{r \in \mathcal{I}_{R}}$ and where $\mathrm{e}^{-2 \jmath \pi \frac{k n^{\prime}}{K}}$ represents (up to the unknown complex factor $\gamma_{i r}$ ) the ideal contribution of a path with a sample shift $n^{\prime}$ in the Doppler channel frequency response associated to the Doppler shift $f$ (see (3) replacing $\tau_{i}$ by $\left.n^{\prime} \frac{T_{u}}{K}\right)$.

The spatial filter $\mathbf{w}$ optimizing $\varepsilon^{2}$ in (10) is given by

$$
\mathbf{w}\left[n^{\prime}, f\right]=\boldsymbol{\Gamma}_{H^{f}}^{-1} \mathbf{h}^{f}\left[n^{\prime}\right]
$$

where the $R \times R$ matrix

$$
\boldsymbol{\Gamma}_{H^{f}} \triangleq \frac{1}{K} \sum_{k \in \mathcal{I}_{K}} \mathbf{H}^{f}[k]\left(\mathbf{H}^{f}[k]\right)^{H}
$$

is an estimate of the spatial covariance matrix of the different frequency responses, and where $\mathbf{h}^{f}\left[n^{\prime}\right]=\left(\hat{h}_{r}^{f}\left[n^{\prime}\right]\right)_{r \in \mathcal{I}_{R}}$.

The output $\mathcal{C}_{\mathrm{ST}}\left[n^{\prime}, f\right]$ of the space-time CHAD is finally obtained computing the energy of the spatially filtered Doppler channel

$$
\mathcal{C}_{\text {ST }}\left[n^{\prime}, f\right]=\left(\mathbf{h}^{f}\left[n^{\prime}\right]\right)^{H} \boldsymbol{\Gamma}_{H^{f}}^{-1} \mathbf{h}^{f}\left[n^{\prime}\right] .
$$


Radar relative bistatic range limitation: for simplicity the previous theoretical developments have been written considering relative propagation delays $\tau_{i}$ less than the guard interval duration $T_{c p}$. It corresponds to a $70 \mathrm{~km}$ relative bistatic coverage for the largest guard interval duration in European DVB-T emissions and to $8.75 \mathrm{~km}$ for the shortest one. Now, it has been stated in [18] that this limitation can reasonably be overridden in return for a gradual detection loss due to the proportion of samples outside the guard interval. Note that this is the case for the experimental results at the end of this paper where relative bistatic delays up to three time the guard interval duration are explored. Moreover this only represents a relative coverage and the LOS distance must be added to obtain the absolute coverage.

\section{Proposed B-STAP DETECTOR}

\section{A. CHAD frequency datacube}

Observing (4), it appears that, for a given array-element $r$, the $M$ instantaneous frequency responses $\left(H_{m r}^{f_{j}}[k]\right)_{k \in \mathcal{E}_{K}}$ can be seen as snapshots of a sinusoidal plane wave impinging a $M$-elements temporal uniform linear array (ULA). The wave being "spatially" characterized by its Doppler shift $f_{j}$. This array-processing analogy can be extended when all the $R$ antennas of the receive array are considered to form a set of $R$ temporal ULAs with $M$ elements each. In that case, the unknown coefficients $\gamma_{i r}$ affect the $M R$-elements antenna global manifold.

Now, using (6) and (7), the expression of the estimated $f$ Doppler channel (8) can be rewritten as

$$
\hat{H}_{r}^{f}[k]=\frac{1}{M} \sum_{m \in \mathcal{I}_{M}} \hat{H}_{m r}[k] \mathrm{e}^{-2 \jmath \pi f m T_{s},}, k_{s} \in \mathcal{E}_{K},
$$

where the instantaneous frequency responses $\left(\hat{H}_{m r}[k]\right)_{k \in \mathcal{E}_{K}}$ are obtained using (7) with no Doppler compensation (i.e. by taking $f=0$ ).

This set of $M R K$ samples $\hat{H}_{m r}[k]$ forms the frequency datacube used by CHAD. For each element $r$, the CHAD processor can then be interpreted as a conventional phaseand-sum beamformer performing on the associated temporal ULA $r$. It aims to enhance the selected $f$-Doppler channel by focusing towards its Doppler direction.

Building on this reinterpretation of CHAD, we propose two main improvements.

A first improvement concerns the choice of the beamformer associated to the $R$ temporal ULAs. CHAD uses a classical data-independent beamformer which only maximizes the signal to noise ratio (SNR). In order to maximize SINR, we then propose to replace the classical beamformer by a linearly constrained minimum variance (LCMV) adaptive (data-dependent) beamformer.

A second improvement concerns the way that the $R$ temporal ULAs are used. With CHAD, the Doppler beamformer does not exploit the spatial diversity brought by the coefficients $\gamma_{i r}$. Time and space dimensions are indeed processed independently: the time dimension only concerns the temporal beamforming while the space dimension appears with the LS spatial filtering. To improve the performance, we then propose to jointly use the temporal and spatial elements to build a space-time adaptive filter.

\section{B. Space-time adaptive SINR maximization}

Let us assume the potential presence of a propagation path with the bistatic delay $\tau_{n^{\prime}}=n^{\prime} T_{u} / K$ and the Doppler shift $f$. For each array-element $r$ and each received pulse $m$, its theoretical contribution to the instantaneous frequency response of the $f$-Doppler channel can be modeled using (4) by

$$
H_{m r}^{f}[k]=\gamma_{r} \mathrm{e}^{j 2 \pi f m T_{s}} \mathrm{e}^{-2 \jmath \pi \frac{k n^{\prime}}{K}}, k \in \mathcal{E}_{K} .
$$

Referring again to the notion of temporal ULA, the sequence of space-time coefficients $\left(\gamma_{r} \mathrm{e}^{\jmath 2 \pi f m T_{s}}\right)_{\substack{r \in \mathcal{I}_{R} \\ m \in \mathcal{T}}}$ can be seen as a space-time array manifold. The $\stackrel{m \in \mathcal{I}^{M}}{M}$ coefficients $u_{m}^{f} \triangleq \mathrm{e}^{j 2 \pi f m T_{s}}$ characterize the Doppler direction of the sinusoidal plane wave $\mathrm{e}^{-2 \jmath \pi \frac{k n^{\prime}}{K}}$ while the $R$ unknown coefficients $\gamma_{r}$ correspond to its spatial direction.

Let us consider now the $M R K$ samples of the frequency datacube $\hat{H}_{m r}[k]$. By linearly combining them, one defines a space-time filtered frequency response:

$$
Z[k]=\sum_{m \in \mathcal{I}_{M}} \sum_{r \in \mathcal{I}_{R}} w_{m r}^{*} \hat{H}_{m r}[k]
$$

where $w_{m r}, m \in \mathcal{I}_{M}, r \in \mathcal{I}_{R}$ are the $M R$ coefficients of the space-time filter. Using array-processing vocabulary, our SINR maximization objective can be formulated as follows: steering to the Doppler direction $f$, the output samples $Z[k]$ have to "fit at best" the theoretical samples $s_{n^{\prime}}[k] \triangleq \mathrm{e}^{-2 \jmath \pi \frac{k n^{\prime}}{K}}$ characterizing the expected plane wave while minimizing the energy of contributions coming with other Doppler directions. That multi-objective problem shall be achieved jointly solving two coupled adaptive optimization schemes: a problem of linearly constrained minimum variance (LCMV) space-time adaptive beamforming combined to a problem of LS spatial adaptive filtering.

1) $L C M V$ adaptive beamformer: the energy $e(w)$ at filter output is given by

$$
\begin{aligned}
e(w) & =\frac{1}{K} \sum_{k \in \mathcal{I}_{K}} Z[k] Z^{*}[k] \\
& =\frac{1}{K} \sum_{m, m^{\prime}, r, r^{\prime}} w_{m r}^{*} w_{m^{\prime} r^{\prime}} \sum_{k} \hat{H}_{m r}[k]\left(\hat{H}_{m^{\prime} r^{\prime}}[k]\right)^{*} .
\end{aligned}
$$

For a plane wave with a Doppler direction $f$, the complex magnitude gain $g_{r}^{f}$ brought by the temporal part $\left(w_{m r}\right)_{m \in \mathcal{I}_{M}}$ of the filter associated to the temporal ULA $r$ yields

$$
g_{r}^{f}=\sum_{m \in \mathcal{I}_{M}}\left(w_{m r}\right)^{*} u_{m}^{f}, \quad \forall r \in \mathcal{I}_{R}
$$


The LCMV formulation of our problem is then to find the optimal coefficients $\left(w_{m r}^{\mathrm{opt}}\right)_{\substack{m \in \mathcal{I}_{M} \\ r \in \mathcal{I}_{R}}}$ minimizing (12) with respect to the $R$ linear temporal steering constraints (13). The necessary knowledge of the gains $g_{r}^{f}$ is here relaxed; they will be determined later during the LS filtering process. One can then write the following constrained optimization problem

$$
\begin{aligned}
& \left(w_{m r}^{\mathrm{opt}}\right)_{\substack{m \in \mathcal{I}_{M} \\
r \in \mathcal{I}_{R}}}= \\
& \underset{\left(w_{m r}\right)_{m r}}{\operatorname{argmin}}\left\{\frac{1}{K} \sum_{m, m^{\prime}, r, r^{\prime}} w_{m r}^{*} w_{m^{\prime} r^{\prime}} \sum_{k} \hat{H}_{m r}[k] \hat{H}_{m^{\prime} r^{\prime}}^{*}[k]\right. \\
& \left.+\sum_{r \in \mathcal{I}_{R}} \mu_{r}\left(g_{r}^{f}-\sum_{m \in \mathcal{I}_{M}} w_{m r}^{*} u_{m}^{f}\right)\right\},
\end{aligned}
$$

where the coefficients $\mu_{r}$ are the $R$ Lagrange multipliers associated to the $R$ constraints.

Using the column stacking operator vec[.], the previous equation can be rewritten in matrix-vector notation. We then define the following column vectors and matrix:

the $M R$-dimensional vector $\mathbf{H}[k]=\operatorname{vec}\left[\left(\hat{H}_{m r}[k]\right)_{\substack{m \in \mathcal{I}_{M} \\ r \in \mathcal{I}}}\right]$ gathering the instantaneous frequency responses, the ${ }^{r \in \mathcal{I}_{R}} R$ vector $\mathbf{w}=\operatorname{vec}\left[\left(w_{m, r}\right)_{\substack{m \in \mathcal{I}_{M} \\ r \in \mathcal{I}_{R}}}\right]$ of the filter coefficients, the $R$-vector of the complex gains $\mathbf{g}_{f}=\left(g_{r}^{f}\right)_{r \in \mathcal{I}_{R}}$, the $M R \times R$ matrix of constraints $\mathbf{C}_{f}=\left[\begin{array}{ccc}\mathbf{u}_{f} & & \mathbf{0} \\ \vdots & \ddots & \vdots \\ \mathbf{0} & & \mathbf{u}_{f}\end{array}\right]$ where $\mathbf{u}_{f}=\left(u_{m}^{f}\right)_{m \in \mathcal{I}}$ is the $M$-dimensional steering vector corresponding to the Doppler direction $f$ and finally $\mu_{f}=\left(\mu_{r}^{f}\right)_{r \in \mathcal{I}_{R}}$, the vector of the $R$ Lagrange multipliers.

With these notations, (14) becomes

$$
\mathbf{w}^{\mathrm{opt}}=\underset{\mathbf{w}}{\operatorname{argmin}}\left\{\mathbf{w}^{H} \boldsymbol{\Gamma} \mathbf{w}+\left(\mathbf{g}_{f}^{H}-\mathbf{w}^{H} \mathbf{C}_{f}\right) \boldsymbol{\mu}_{f}\right\},
$$

where

$$
\Gamma=\frac{1}{K} \sum_{k} \mathbf{H}[k] \mathbf{H}^{H}[k],
$$

is an estimate of the Hermitian $(M R \times M R)$ space-time covariance matrix. Nulling the gradient of the Lagrangian in (15) with respect to (w.r.t.) $\mathbf{w}^{H}$, it comes

$$
\mathbf{w}^{\mathrm{opt}}=\boldsymbol{\Gamma}^{-1} \mathbf{C}_{f} \boldsymbol{\mu}_{f} .
$$

Now, replacing the expression (16) of $\mathbf{w}$ in the linear system of constraints (13), $\mathbf{g}_{f}^{H}=\mathbf{w}^{H} \mathbf{C}_{f}$, we get the Lagrange multiplier vector:

$$
\boldsymbol{\mu}_{f}=\left(\mathbf{C}_{f}^{H} \boldsymbol{\Gamma}^{-1} \mathbf{C}_{f}\right)^{-1} \mathbf{g}_{f} .
$$

The general expression of the space-time filter (16) w.r.t. the unknown spatial complex gains then yields

$$
\mathbf{w}^{\mathrm{opt}}=\mathbf{M g}_{f},
$$

where $\mathbf{M}$ is the following $(M R \times R)$ rectangular matrix

$$
\mathbf{M}=\boldsymbol{\Gamma}^{-1} \mathbf{C}_{f}\left(\mathbf{C}_{f}^{H} \boldsymbol{\Gamma}^{-1} \mathbf{C}_{f}\right)^{-1} \text {. }
$$

2) LS spatial adaptive filtering: we can now set the optimal complex gain vector $\mathbf{g}^{\text {opt }}$ so that the samples $Z[k]$ fit those of the sinusoidal plane wave $s_{n^{\prime}}[k]=\mathrm{e}^{-2 \jmath \pi \frac{k n^{\prime}}{K}}$ in the LS sense:

$$
\mathbf{w}^{\mathrm{opt}}=\underset{\mathbf{w}}{\operatorname{argmin}}\left\{\frac{1}{K} \sum_{k \in \mathcal{I}_{K}}\left|\mathbf{w}^{H} \mathbf{H}[k]-s_{n^{\prime}}[k]\right|^{2}\right\} .
$$

Replacing $\mathbf{w}$ using (17), an equivalent formulation is

$$
\begin{aligned}
\mathbf{g}^{\text {opt }} & =\underset{\mathbf{g}_{f}}{\operatorname{argmin}} \sum_{k \in \mathcal{I}_{K}} \frac{1}{K}\left|\mathbf{g}_{f}^{H} \mathbf{M}^{H} \mathbf{H}[k]-s_{n^{\prime}}[k]\right|^{2} \\
& =\underset{\mathbf{g}_{f}}{\operatorname{argmin}}\left\{\mathbf{g}_{f}^{H} \mathbf{M}^{H} \mathbf{\Gamma} \mathbf{M g}_{f}-\mathbf{g}_{f}^{H} \mathbf{M}^{H} \frac{1}{K} \sum_{k \in \mathcal{I}_{K}}\left(\mathbf{H}[k] s_{n^{\prime}}^{*}[k]\right)\right. \\
& \left.-\frac{1}{K} \sum_{k \in \mathcal{I}_{K}}\left(s_{n^{\prime}}[k] \mathbf{H}^{H}[k]\right) \mathbf{M g}_{f}+\frac{1}{K} \sum_{k \in \mathcal{I}_{K}}\left|s_{n^{\prime}}[k]\right|^{2}\right\} .
\end{aligned}
$$

Nulling the gradient of the mean square error w.r.t. $\mathbf{g}_{f}^{H}$, we get

$$
\mathbf{g}^{\mathrm{opt}}=\left(\mathbf{M}^{H} \mathbf{\Gamma} \mathbf{M}\right)^{-1} \mathbf{M}^{H} \frac{1}{K} \sum_{k \in \mathcal{I}_{K}}\left(\mathbf{H}[k] s_{n^{\prime}}^{*}[k]\right) .
$$

Replacing the previous expression of $\mathbf{g}^{\text {opt }}$ in (17), it comes

$$
\mathbf{w}^{\text {opt }}=\mathbf{M}\left(\mathbf{M}^{H} \boldsymbol{\Gamma M}\right)^{-1} \mathbf{M}^{H} \frac{1}{K} \sum_{k \in \mathcal{I}_{K}}\left(\mathbf{H}[k] s_{n^{\prime}}^{*}[k]\right) .
$$

Now, since $s_{n^{\prime}}^{*}[k]=\mathrm{e}^{2 \jmath \pi \frac{k n^{\prime}}{K}}$, it appears that the expression $\frac{1}{K} \sum_{k \in \mathcal{I}_{K}} \mathbf{H}[k] s_{n^{\prime}}^{*}[k]$ stands for the IDFT of $(\mathbf{H}[k])_{k \in \mathcal{I}_{K}}$, denoted by $\left(\mathbf{h}\left[n^{\prime}\right]\right)_{n^{\prime} \in \mathcal{I}_{K}}$. Finally, using (18), we get

$$
\mathbf{w}^{\mathrm{opt}}\left[n^{\prime}, f\right]=\boldsymbol{\Gamma}^{-1} \mathbf{C}_{f}\left(\mathbf{C}_{f}^{H} \boldsymbol{\Gamma}^{-1} \mathbf{C}_{f}\right)^{-1} \mathbf{C}_{f}^{H} \boldsymbol{\Gamma}^{-1} \mathbf{h}\left[n^{\prime}\right]
$$

The corresponding space-time adaptive detector $\mathcal{C}_{\mathrm{B}-\mathrm{STAP}}\left[n^{\prime}, f\right]$ is finally built computing the energy $\frac{1}{K} \sum_{k}|\mathbf{Z}[k]|^{2}$ at the filter output. It straightforwardly comes using $\mathbf{Z}[k]=\left(\mathbf{w}^{\mathrm{opt}}\right)^{H} \mathbf{H}[k]$ with (21):

$$
\mathcal{C}_{\mathrm{B}-\mathrm{STAP}}\left[n^{\prime}, f\right]=\mathbf{h}^{H}\left[n^{\prime}\right] \boldsymbol{\Gamma}^{-1} \mathbf{C}_{f}\left(\mathbf{C}_{f}^{H} \boldsymbol{\Gamma}^{-1} \mathbf{C}_{f}\right)^{-1} \mathbf{C}_{f}^{H} \boldsymbol{\Gamma}^{-1} \mathbf{h}\left[n^{\prime}\right] .
$$

\section{Computational load}

Pilot-aided synchronization and OFDM symbol detection are two essential prior steps for all the methods to provide an ideal noise-free reference signal. The computational load of these two steps remains very low since it simply corresponds to a classical DVB-T decoding: synchronization by correlation with the pilot sequence can be performed only once using a few symbols. Then, extraction of the sequence of $M$ OFDM symbols using $K$-points FFT leads to a linear complexity: $\mathcal{O}\left(M K \log _{2} K\right)$.

Let us put it in the extreme case where one wants to scan $K$ bistatic delays $\tau_{n^{\prime}}$ and then to compute, for a given Doppler shift $f$, the $(K)$-vector $\left.\left(\mathcal{C}_{\mathrm{STAP}}\left[n^{\prime}, f\right]\right)\right)_{n^{\prime} \in \mathcal{I}_{K}}$.

In the expression (22), the $K(M R)$-vectors $\boldsymbol{\Gamma}^{-1} \mathbf{h}\left[n^{\prime}\right]$ do not depend on the Doppler frequency shift under test $f$ and are then calculated only once. Since a $K$-points fast Fourier transform (FFT) can be used to efficiently compute $\mathbf{h}\left[n^{\prime}\right]$ 
from $\mathbf{H}[k]$, the computational load remains dominated by the inversion of the $(M R \times M R)$ matrix $\Gamma$ and one can then expect a computational complexity bounded by $\mathcal{O}\left(M^{3} R^{3}\right)$. Note that $K$ space-time matrices should have to be inverted using SMI algorithm, increasing the computational load to $\mathcal{O}\left(K M^{3} R^{3}\right)$.

For each value of the Doppler frequency shift $f$, one first has to compute the $K(R)$-vectors $\left(\mathbf{C}_{f}^{H} \boldsymbol{\Gamma}^{-1} \mathbf{h}\left[n^{\prime}\right]\right)_{n^{\prime} \in \mathcal{I}_{K}}\left(M R^{2} K\right.$ complex operations) and the $(R \times R)$-matrix $\left(\mathbf{C}_{f}^{H} \boldsymbol{\Gamma}^{-1} \mathbf{C}_{f}\right)^{-1}$ $\left(2 M^{2} R^{3}+R^{3}\right.$ complex operations). Finally, $K\left(R^{2}+R\right)$ complex operations are still needed to complete calculation of the $(K)$-vector $\left.\left(\mathcal{C}_{\mathrm{STAP}}\left[n^{\prime}, f\right]\right)\right)_{n^{\prime} \in \mathcal{I}_{K}}$. The total number of operations is then $M R^{2} K+2 M^{2} R^{3}+R^{3}+K\left(R^{2}+R\right)$ i.e. a $\mathcal{O}\left(M R^{2} K\right)$ computational complexity .

By contrast, efficiently computing the cross-ambiguity function in the frequency domain, using the FFT, one obtains the complexity $\mathcal{O}\left(M R K \log _{2} K\right)$ for each frequency shift $f$. Thus, with e.g. $K=8192$ orthogonal subcarriers, the proposed detector (B-STAP CHAD) has a lower computational cost than CAF if the number $R$ of antennas is less than $\log _{2}(K)=\log _{2}(8192)=13$.

\section{TARGET RETURNS FROM EXPERIMENTAL DATA}

We dispose of two experimental datasets delivered by two prototypes of passive ground radar. The first dataset referred as Dataset \#1, is provided by a 4-elements ULA. The target of interest is an helicopter illuminated by the French DVB-T transmitter from the Eiffel tower during the french Bastille day. The second dataset (Dataset \#2) comes from a second prototype using a 16-elements $2 \mathrm{D}$ planar receive array. One can learn more about this second system by reading [22], [23]. The objective is here to discriminate a fighter aircraft mainly illuminated by three German DVB-T transmitters using the same HF carrier frequency (single frequency network, SFN).

\section{A. Dataset \#1 (helicopter-4-elements ULA):}

The characteristics of the DVB-T emission are $8 \mathrm{~K}$ ( $K=8192$ orthogonal subcarriers), 64-QAM and $T_{c p}=T_{u} / 32$. All detection results are obtained processing the same sequence of $M=190$ consecutive OFDM symbols (pulses) corresponding to an observation duration of approximatively $170 \mathrm{~ms}$. The reference signal is extracted from a dedicated DVB-T directive antenna pointing towards the Eiffel tower.

In the sequel, relative bistatic distances up to $25 \mathrm{~km}$ are scanned corresponding to 3 times the distance covered by the wave during the guard interval duration. In the range-Doppler plane, the theoretical location of the target echo of interest $(17.1 \mathrm{~km},-173.0 \mathrm{~Hz})$ is marked by a black circle. Such a range value corresponds to a relative bistatic delay more than twice as large as the $\mathrm{CP}$ duration and a slight discrimination loss (about $10 \log _{10}\left((32-2)^{2} / 32^{2}\right)=-0.57 d B$, see [18]) is expected using CHAD with respect to CAF.

To tackle dynamic range issues between the different target returns, we adjusted, in each following 3D surveillance map, the colorscale so that the highest peak with a unit normalized level, appears with a black color while the clutter floor has always the same gray/blue color. In that way, whatever the process used, a detection peak is equally (and fairly) visible depending on its relative level wrt the clutter floor.

The figure 2 shows first target returns obtained using CAF (5) without rejecting the zero-Doppler contribution. Except

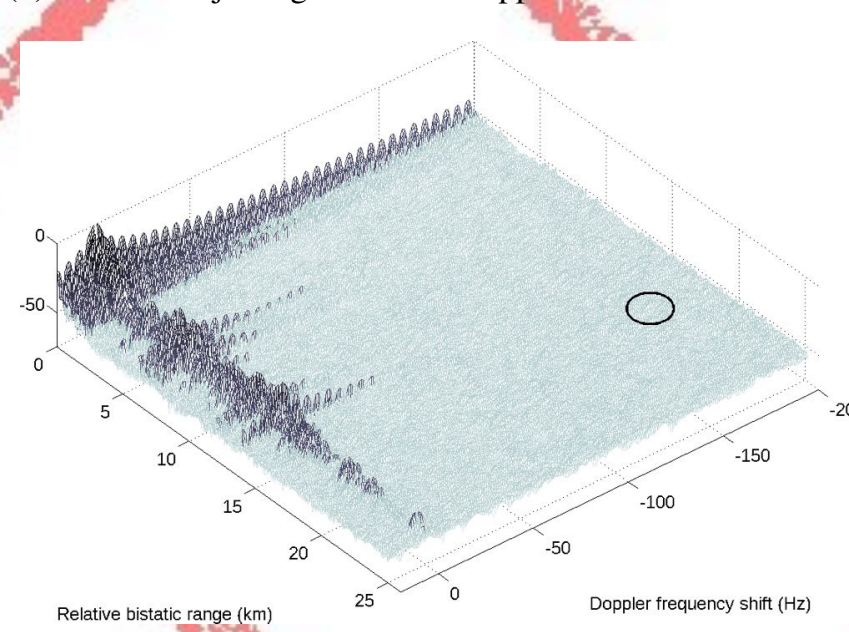

Fig. 2. Space-time CAF $\mathcal{A}_{\mathrm{ST}}(\mathrm{dB})$ without $\mathrm{ZDC}$ rejection.

for the dominant LOS signal and for some other zero-Doppler returns, no moving target is visible. They are all masked by the clutter floor high level mainly induced by the LOS path.

The next result (Fig. 3) is obtained using space-time CHAD (11) without ZDC rejection. The clutter floor level being here

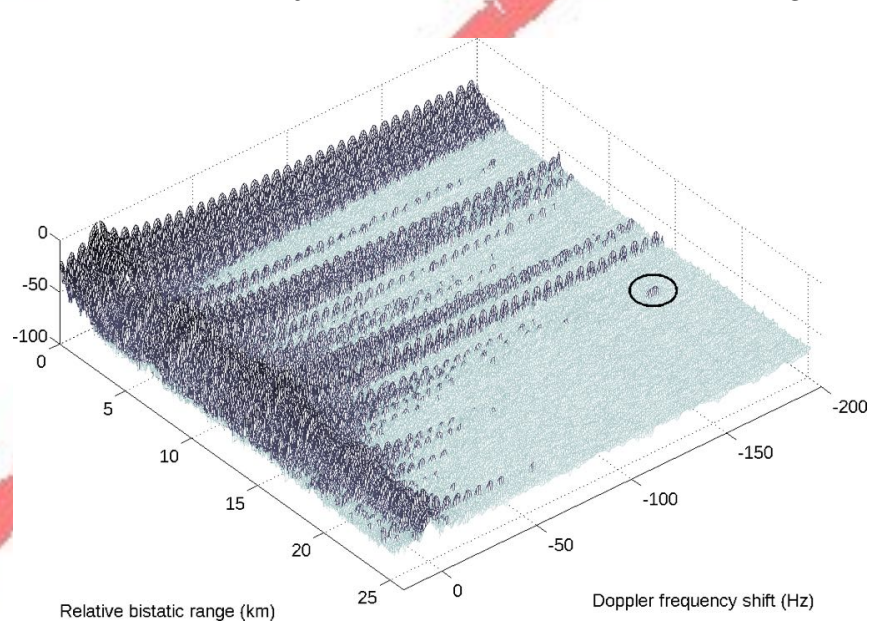

Fig. 3. Space-time $\mathrm{CHAD} \mathcal{C}_{\mathrm{ST}}(\mathrm{dB})$ without $\mathrm{ZDC}$ rejection.

lower than using CAF, more important ZDC leakages appear in the detection map. These secondary lobes nevertheless do not mask the target of interest which is now visible inside the black circle.

A cut along the Doppler shift of the target of interest (-173 $\mathrm{Hz}$ ) is presented in the upper-bound normalized plot of Fig. 4 for each of the two space-time detectors. It appears that 


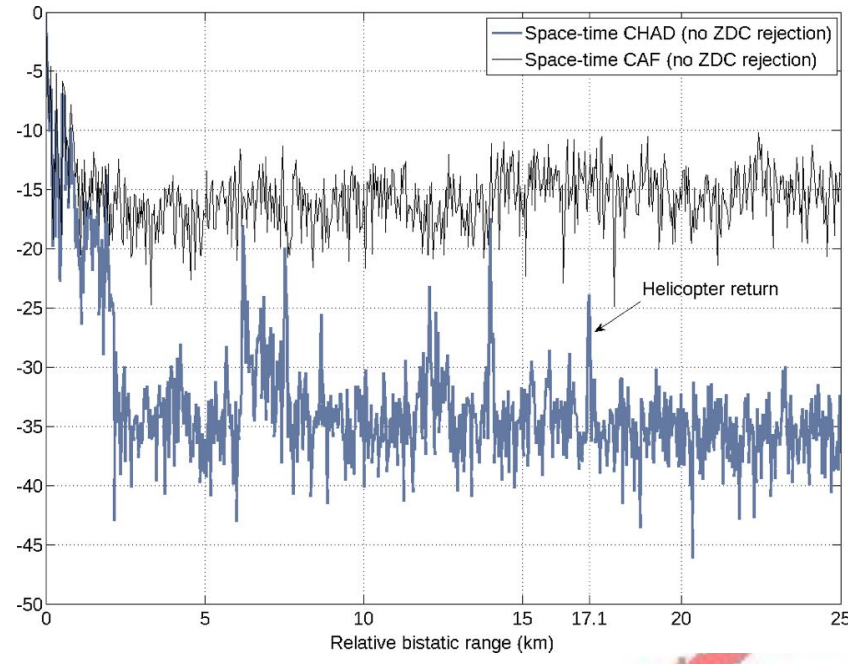

Fig. 4. $\mathcal{A}_{\mathrm{ST}}$ vs $\mathcal{C}_{\mathrm{ST}}(\mathrm{dB})$, both without $\mathrm{ZDC}$ rejection - range values.

the clutter level reduction brought by $\mathrm{CHAD}$ reaches about $20 \mathrm{~dB}$, allowing to bare the helicopter return.

Fig. 5 corresponds to target returns from CAF implementing the ZDC rejection frequency method proposed in [13]. As expected, one can see that fully ZDC rejection is not

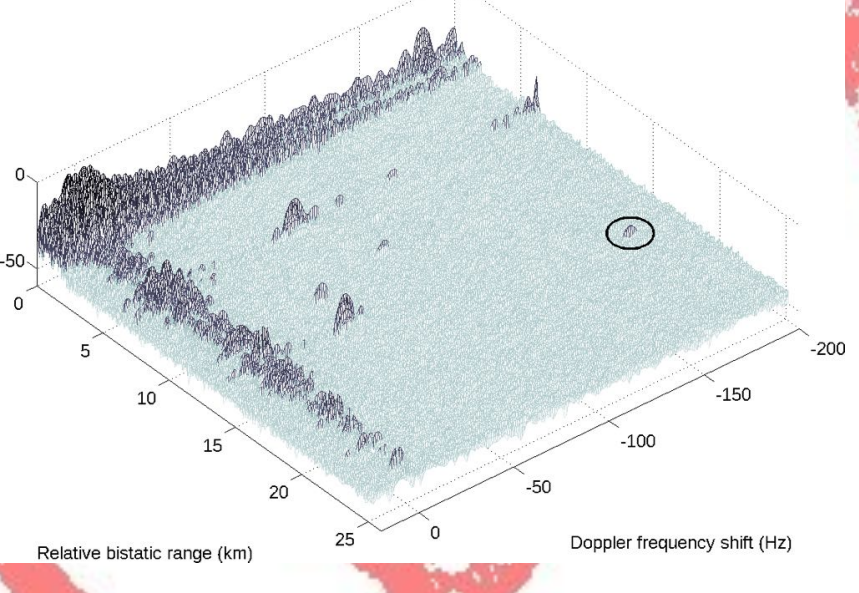

Fig. 5. Space-time CAF $\mathcal{A}_{\mathrm{ST}}(\mathrm{dB})$ with ZDC rejection.

reached and quite important secondary lobes remain along the Doppler axis in particular for low bistatic ranges. This could be caused by RF impairments (residual CFO) or mechanical impairments (low frequency vibrations of the antenna support). The ZDC rejection level is nevertheless sufficient to unmask several target returns including that of interest within the black circle.

A similar result (see Fig. 6) is obtained using space-time CHAD implementing the embedded ZDC rejection method proposed in [13]. The range values (from $15 \mathrm{~km}$ to $19 \mathrm{~km}$, i.e. around the target-of-interest location) in Fig. 7 show that after ZDC rejection, the two detectors exhibit close clutter relative levels.

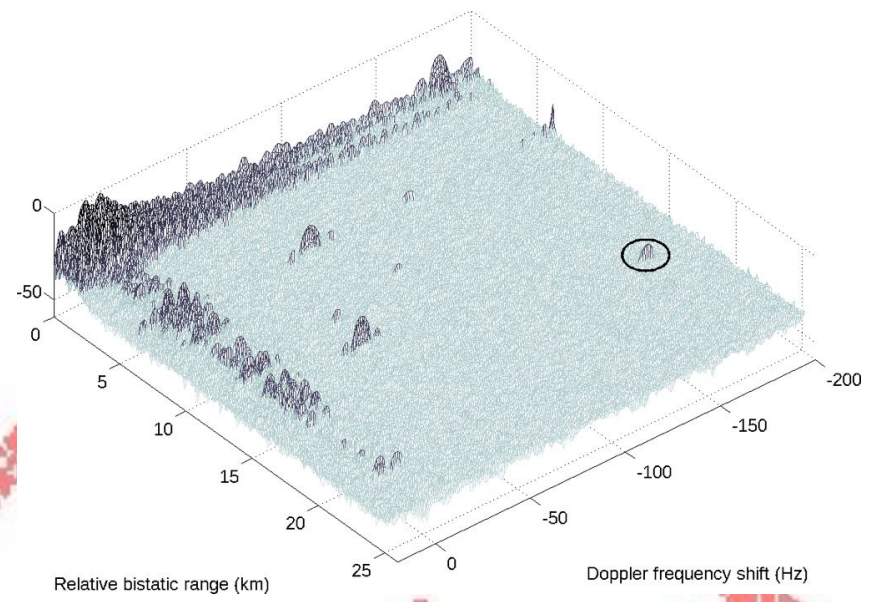

Fig. 6. Space-time CHAD $\mathcal{C}_{\mathrm{ST}}(\mathrm{dB})$ with $\mathrm{ZDC}$ rejection. 


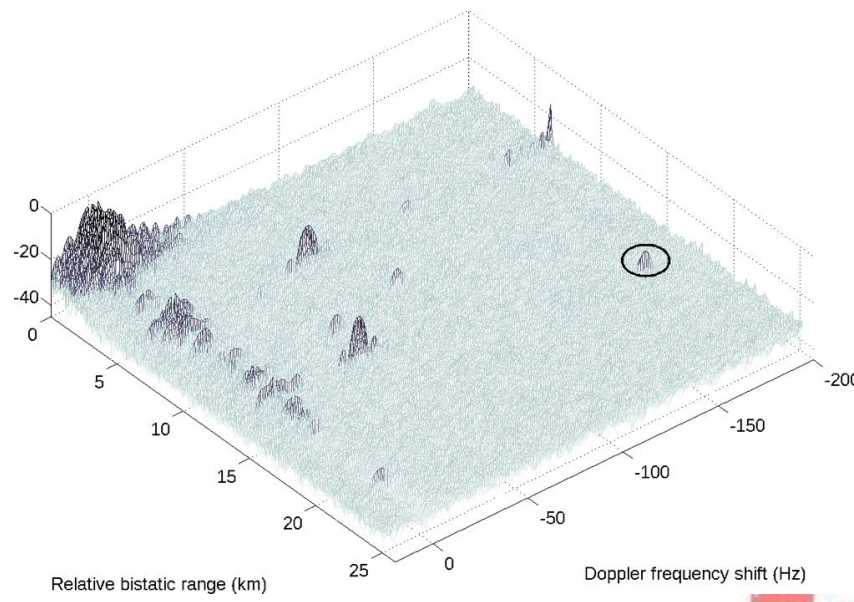

Fig. 8. Blind space-time adaptive $\mathrm{CHAD} \mathcal{C}_{\mathrm{B}-\mathrm{STAP}}(\mathrm{dB})$.

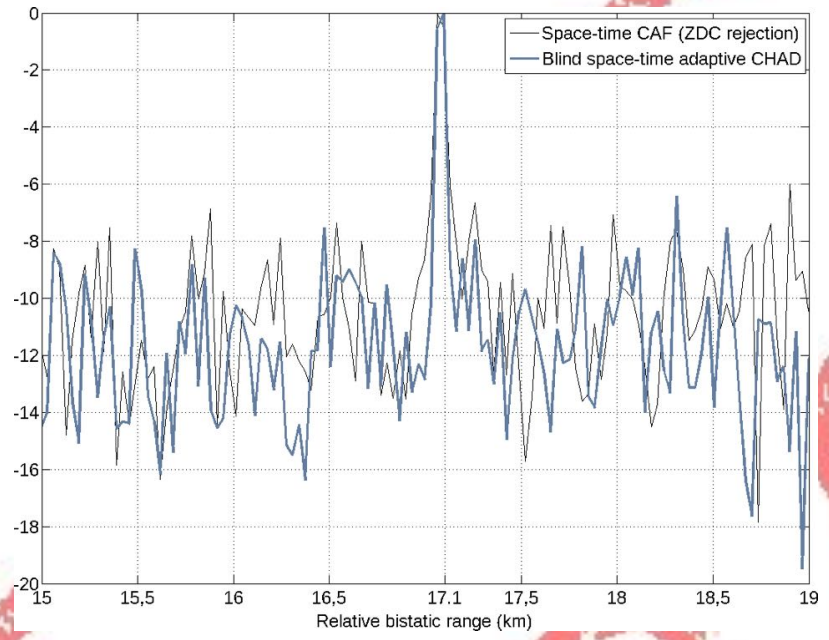

Fig. 9. $\mathcal{A}_{\mathrm{ST}}(\mathrm{dB})$ with $\mathrm{ZDC}$ rejection $v s \mathcal{C}_{\mathrm{B}-\mathrm{STAP}}(\mathrm{dB})$ - range values

signal is obtained from the radar receive array-antenna using the spatial approach proposed in [21]. The 16 recordings are synchronized to the LOS path of the nearest transmitter. A bistatic coverage up to about $140 \mathrm{~km}$ is explored. It corresponds to bistatic delays up to 2 times the guard interval duration.

The target of interest (the fighter aircraft) being illuminated by three DVB-T transmitters (SFN configuration), three returns are expected in the range-Doppler plane with the following coordinates: $(8.1 \mathrm{~km},-338 \mathrm{~Hz}),(33.8 \mathrm{~km},-249 \mathrm{~Hz})$ and $(76.8 \mathrm{~km},-123 \mathrm{~Hz})$. These theoretical locations will be marked by circles in the surveillance maps. Note that the aircraft echo with the highest range coordinate $(76.8 \mathrm{~km})$ falls outside the guard interval.

A first surveillance map is presented in Fig. 10. It is obtained using CAF without implementing the rejection of the dominant zero-Doppler contribution. One can observe several zero-Doppler powerful returns corresponding to the three LOS signals from the three SFN transmitters and to several strong replicas. In addition, one can see a series of aligned plots with Doppler shifts of about $-240 \mathrm{~Hz}$. They are

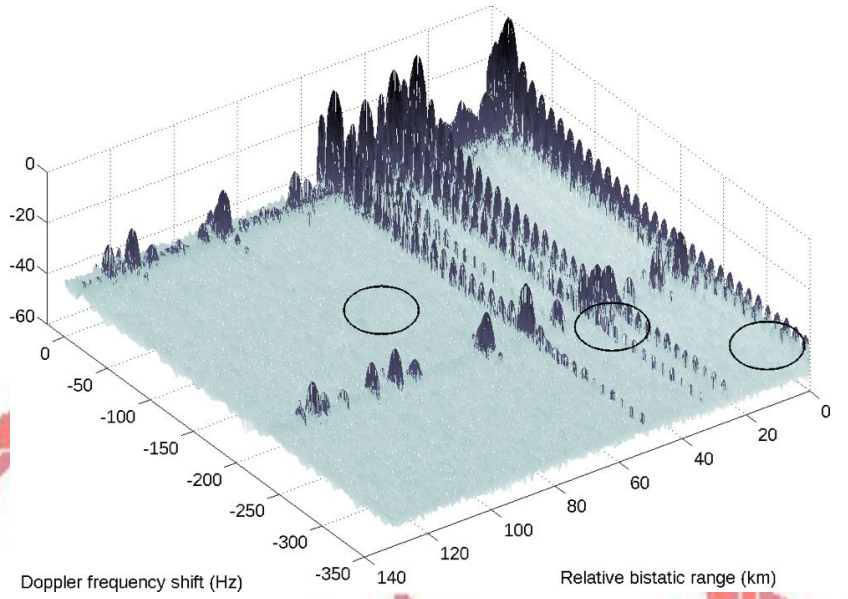

Fig. 10. Space-time CAF $\mathcal{A}_{\mathrm{ST}}(\mathrm{dB})$ without ZDC rejection.

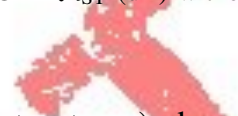

false alarms (ghost returns) due to the particular structure of the DVB-T signal [17]. The target-of-interest returns remain masked by the clutter floor induced by the dominant contribution.

The next figure presents a surveillance map obtained using the space-time extension of CHAD without ZDC rejection.

As with the first dataset, one observes a very important

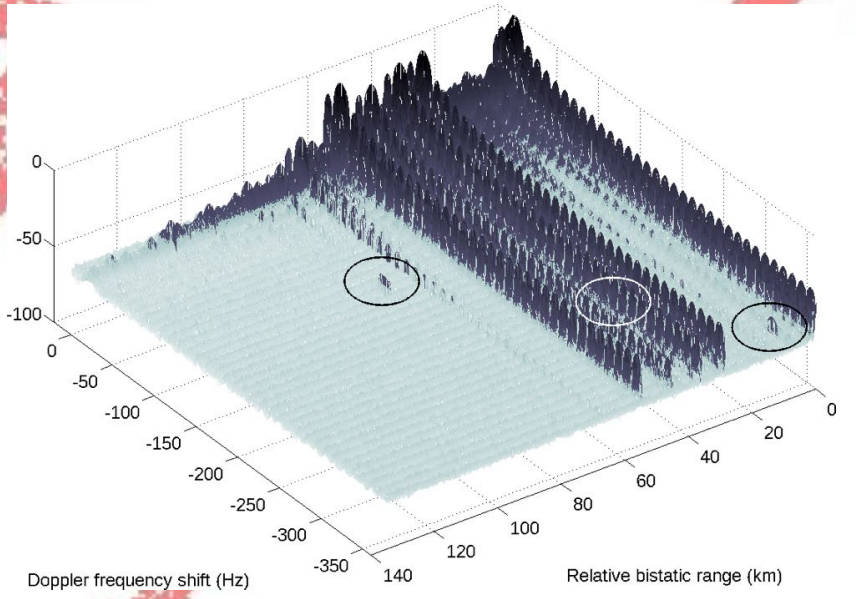

Fig. 11. Space-time $\mathrm{CHAD} \mathcal{C}_{\mathrm{ST}}(\mathrm{dB})$ without $\mathrm{ZDC}$ rejection.

reduction of the clutter floor level, hence two of the three aircraft returns are now visible. The third one (white circle) outreaches the clutter floor but remains hidden between important secondary lobes. As expected with channel-based approaches, the ghost returns no more appear.

The surveillance map in the next figure 12 corresponds to a space-time CAF after ZDC rejection. Again, due to various impairments, disturbing residual leakages from the zero-Doppler contribution can be observed all along the Doppler dimension. Several new target returns are however found and those of the fighter aircraft appear now inside the black circles. One may still note the presence of duplicated ghost peaks caused by the incomplete rejection of ZDC. 


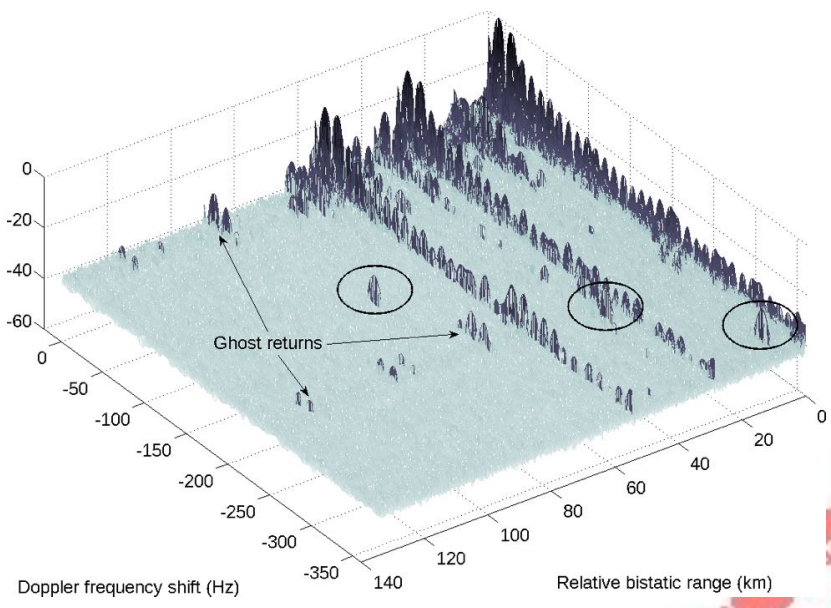

Fig. 12. Space-time CAF $\mathcal{A}_{\mathrm{ST}}(\mathrm{dB})$ with ZDC rejection.

The map presented in the next figure 13 is delivered by space-time CHAD implementing a ZDC rejection. A similar

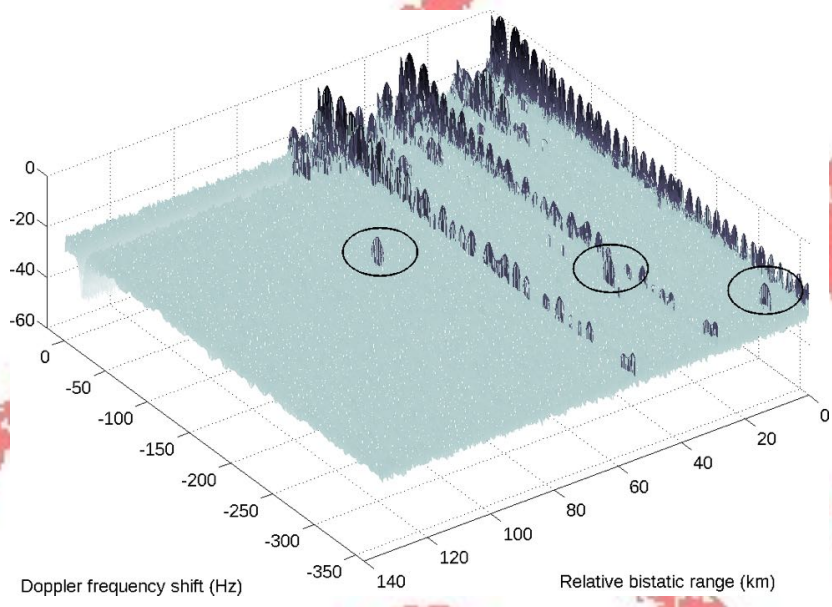

Fig. 13. Space-time $\mathrm{CHAD} \mathcal{C}_{\mathrm{ST}}(\mathrm{dB})$ with ZDC rejection.

performance than with CAF (see previous Fig. 12) is observed, except that the ghost returns are now eliminated.

The following result (Fig. 14) is obtained using the proposed detector $\mathcal{C}_{\mathrm{B}-\mathrm{STAP}}$. As a result of SINR maximization, the three LOS return peaks are now correctly relocated around the zero-Doppler axis hence the surveillance map is globally "cleaner" than that obtained using the space-time detector CAF or even using CHAD. The three aircraft returns are perfectly discriminated wrt the background. In addition, since no ZDC rejection was performed, several slow moving targets are now found. Most of them are due to the presence of a civil airport and an highway within the radar range [22], [23].

Fig. 15 shows the results obtained with both $\mathcal{A}_{\mathrm{ST}}$ (with ZDC rejection) and $\mathcal{C}_{\mathrm{B}-\mathrm{STAP}}$ depending on the bistatic distance, along the $-123 \mathrm{~Hz}$ Doppler frequency shift. It corresponds to the fighter aircraft expected return with the highest bistatic distance $(76.8 \mathrm{~km})$. One can see that, around the

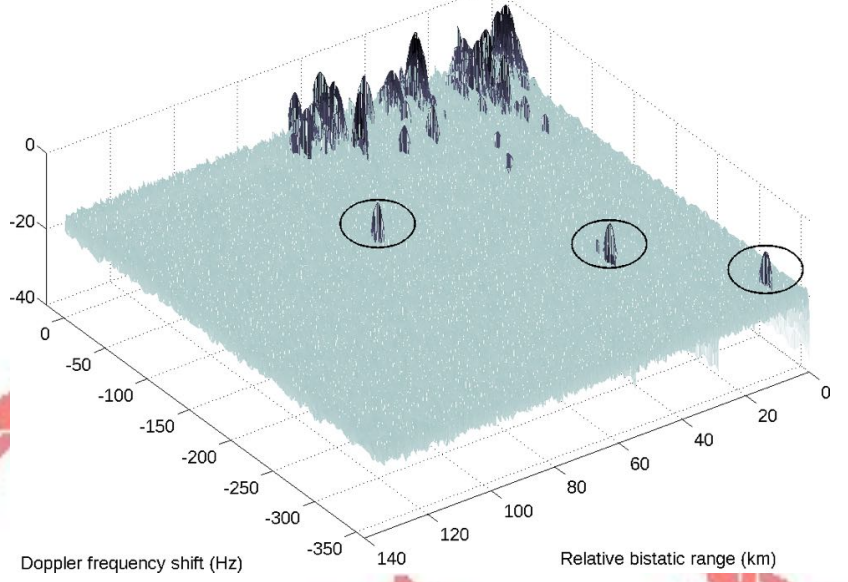

Fig. 14. Blind space-time adaptive $\operatorname{CHAD} \mathcal{C}_{\mathrm{B}-\mathrm{STAP}}(\mathrm{dB})$.

peak of interest, a similar performance is reached by the two processings. One can however observe the ability of B-STAP CHAD to remove secondary lobes; in the figure, a secondary lobe coming from one of the three LOS propagation paths with a relative bistatic distance of about $54.8 \mathrm{~km}$.

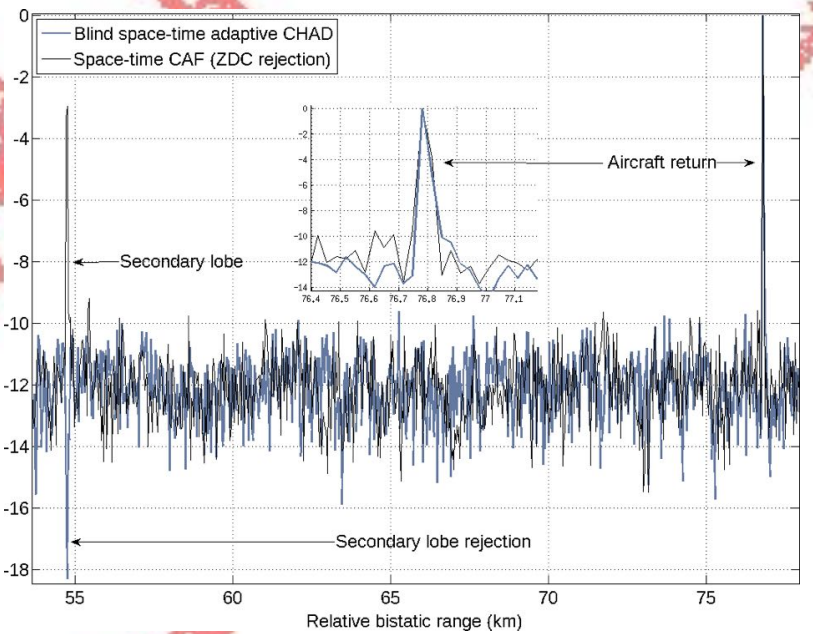

Fig. 15. $\mathcal{A}_{\mathrm{ST}}(\mathrm{dB})$ with $\mathrm{ZDC}$ rejection $v s \mathcal{C}_{\mathrm{B}-\mathrm{STAP}}(\mathrm{dB})-$ range values.

The last two figures Fig. 16 and Fig. 17 present rangeDoppler trackings of one of the three aircraft returns (that illuminated by the nearest DVB-T transmitter). For each method (space-time CAF with ZDC rejection and blind spacetime adaptive CHAD), 12 range-Doppler surveillance maps have been computed from 12 sequences of 82 OFDM pulses each. The sequences being separated by 1 second, we obtain a 12 seconds tracking. These maps were combined (elementwise maximum) then thresholded to produce 2D binary detection maps. All the 12 returns forming a "Z"-shaped track are detected by the two approaches with a similar performance. In addition to the ghost returns suppression, the main difference lies in the ability of the proposed method to work with the dominant interference while with CAF, this disturbance must first be removed. 


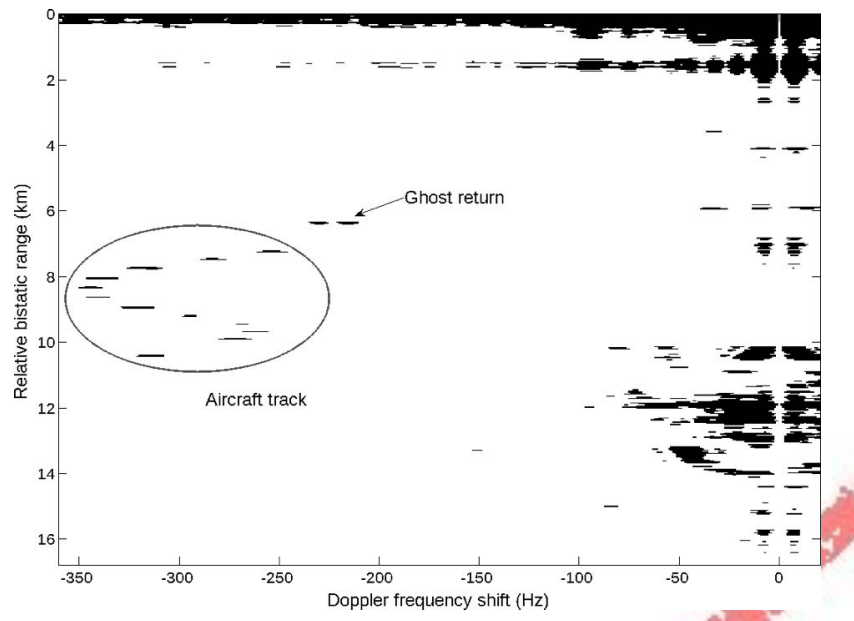

Fig. 16. $\mathrm{ZDC}$ rejection $+\mathcal{A}_{\mathrm{ST}}(\mathrm{dB})-12$ seconds aircraft tracking (2D view).

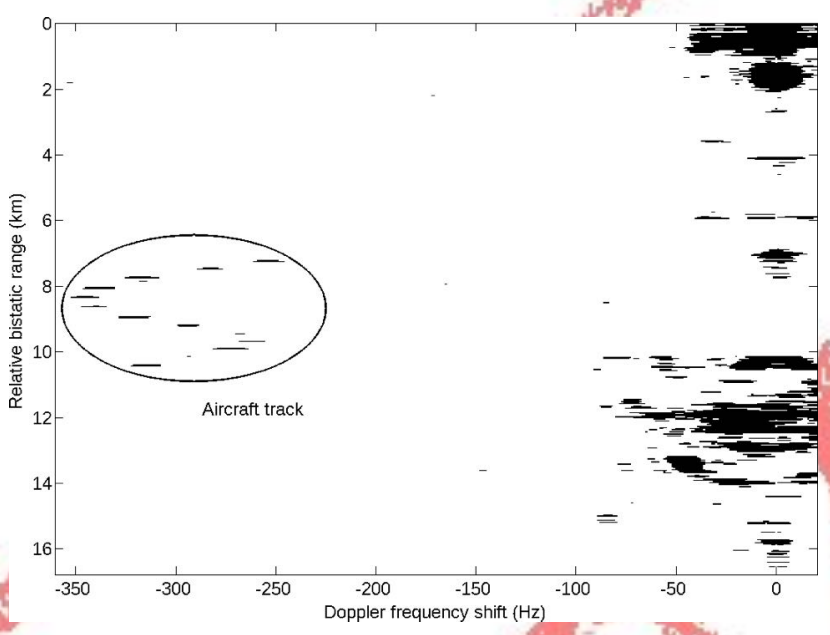

Fig. 17. $\mathcal{C}_{\mathrm{STAP}}(\mathrm{dB})-12$ seconds aircraft tracking (2D view).

\section{CONCLUSION}

In this paper, a new fully adaptive STAP for rangeDoppler detection of targets with ODFM-based passive radars is developed. Unlike classical STAP approaches that perform from matched filtered datacubes, the proposed method uses a channel-based (signal-independent) frequency datacube built on the particular structure of the OFDM pulses. Considering a set of complex exponentials as a new target signal model to be detected in the CHAD datacube, we show that SINR maximization can be reached for the whole set of range-Doppler bins, from a unique space-time sample matrix without using any training data. This explains the very low computational cost of the method. Additionally, since no spatial beamscan is performed, the knowledge of the array shape is not required and the target detection is processed from a single rangeDoppler map. Its use on experimental datasets illustrates the practical interests of such an approach: dominant interference prerejection not required, secondary lobes reduction, ghost returns elimination, detection improvement for weak slow moving targets. The proposed approach dealing intrinsically with a wide range of hardware impairments, it allows to easily implement a performing passive radar system for a low cost. Finally, B-STAP CHAD should be useful for OFDM-based airborne passive radars where the presence of the (generally Dopplerized) dominant interference would no longer be a major issue.

\section{ACKNOWLEDGMENTS}

The campaign of measurements associated to Dataset \#2 was achieved by Thales Air Systems, ONERA, DGA (Direction genérale de l'armement) and CEAM (Centre d'Experiences Aériennes Militaires) jointly with BWB (Bundesamt fur Wehrtechnik und Beschaffung) and the Fraunhofer Institute. We are particularly grateful to J. Colin, from DGA, for giving us access to data.

\section{APPENDIX}

\section{Receive Signal Modeling - Simplifying Assumptions}

The LOS RF complex signal received by the reference antenna-element (indexed by 0 ) is generically denoted by:

$$
\underset{\sim}{s}(t)=s(t) e^{2 \jmath \pi F_{0} t},
$$

where $s(t)$ represents the complex envelop (baseband signal) and $F_{0}$ the HF carrier frequency.

Let us assume that it exists at the bistatic initial distance $d(i, 0)$, a moving reflector $i$ with the constant bistatic speed $v_{i}$.

The corresponding reflected signal impinges the reference element with a LOS relative varying delay $\tau_{i}^{\prime}(t)$. Its contribution to the reference element is then proportional to

$$
\underset{\sim}{s}\left(t-\tau_{i}^{\prime}(t)\right)=s\left(t-\tau_{i}^{\prime}(t)\right) \mathrm{e}^{2 \jmath \pi F_{0}\left(t-\tau_{i}^{\prime}(t)\right)} .
$$

Assume also that the bistatic speed $v_{i}$ is sufficiently low so that the distance covered by the target during the observation time $T$ is negligible with respect to the bistatic distance $d(i, 0)$ :

$$
v_{i} T \ll d(i, 0) \text {. }
$$

This allows to limit the degree of the polynomial expression $\tau_{i}^{\prime}(t)$ to one and we then have

$$
\tau_{i}^{\prime}(t) \approx \frac{d_{i}}{c}+\frac{v_{i}}{c} t, t \in[0, T]
$$

where $d_{i}=d(i, 0)-d(0,0)$ is the bistatic distance relative to the LOS distance and where $c$ is the light speed.

Still assume that the distance covered by the target during the observation time $T$ is inferior to the shortest wavelength in baseband:

$$
v_{i} T \ll \frac{c}{B},
$$

where $B$ is the bandwidth of the signal $s(t)$. One can then ignore the effect of the first order term $\frac{v_{i}}{c} t$ within the complex envelop and write

$$
\underset{\sim}{s}\left(t-\tau_{i}^{\prime}(t)\right) \approx \alpha_{i} s\left(t-\tau_{i}\right) \mathrm{e}^{2 \jmath \pi \mathrm{f}_{i} t} \mathrm{e}^{2 \jmath \pi F_{0} t}, \quad t \in[0, T],
$$

where $\tau_{i}=\frac{d_{i}}{c}, \alpha_{i}=\mathrm{e}^{-2 \jmath \pi F_{0} \tau_{i}}$ and where $\mathrm{f}_{i}=-F_{0} \frac{v_{i}}{c}$ is the Doppler frequency shift.

On any antenna-element $r \neq 0$, the signal is received with an algebraic relative delay denoted by $\tau_{i r}^{\prime}(t)$. Due to the small 
dimension of the array-antenna with respect to the bistatic distances involved, it is reasonable to assume that the speed $v_{i}$ is viewed in the same way by all the elements and this relative delay can be considered as a constant $\left(\tau_{i r}^{\prime}(t) \approx \tau_{i r}\right)$ during the observation time. Additionally, the dimension of the array being very small compared to the shortest wavelength in baseband, one can assume that $B \max _{r}\left[\tau_{i r}\right] \ll 1$ and consequently the effect of $\tau_{i r}$ within the complex envelop can be neglected. It follows that $\forall t \in[0, T]$, the contribution of the reflected signal that impinges the array-element $r$ yields

$$
\alpha_{i} \mathrm{e}^{-2 \jmath \pi F_{0} \tau_{i r}} s\left(t-\tau_{i}\right) \mathrm{e}^{2 \jmath \pi \mathrm{f}_{i} t} \mathrm{e}^{2 \jmath \pi F_{0} t} .
$$

Finally, considering now a general multipath propagation scheme, the whole baseband signal $y_{r}(t)$ received on any element $r$ can then be modeled by a noisy linear combination of the IQ frequency downconverted contributions (23):

$$
y_{r}(t)=\sum_{i \in \mathcal{I}_{I}} \gamma_{i r} s\left(t-\tau_{i}\right) \mathrm{e}^{\jmath 2 \pi \mathrm{f}_{i} t}+\eta_{r}(t), t \in[0, T],
$$

where the noise term $\eta_{r}(t)$ is assumed to be white, Gaussian and statically independent from one element $r$ to another. In practice, the complex coefficients $\gamma_{i r}$ do not exactly match $\alpha_{i} \mathrm{e}^{2 \jmath \pi F_{0} \tau_{i r}}$ since they may include all additional effects modifying the phases and the amplitudes of the different contributions. These effects depend on target radar crosssection, on the complex gain spatial pattern of each antennaelement $r$, and on other unknown impairments such as antenna calibration errors.

\section{REFERENCES}

1] J. Ward, "Space-time adaptive processing for airborne radar," Technical Report 1015, MIT Lincoln Laboratory, Lexington, MA, December 1994

[2] D. K. P. Tan, "Signal Processing for Airborne Passive Radar: Interference Suppression and Space Time Adaptive Processing Techniques for Transmissions of Opportunity”. Ph.D. dissertation, November 2012

[3] X. Neyt, J. J. Raout, M. Kubica, V. Kubica, S. Roques, J. G. Verly, and M. Acheroy, "Feasibility of STAP for passive GSM-based radar," in IEEE Radar Conference, (Verona, NY), pp. 546-551, 24-26 April 2006.

[4] G. Fabrizio, F. Colone, P. Lombardo, and A. Farina, "Adaptive beamforming for high-frequency over-the-horizon passive radar," IET Radar, Sonar and Navigation, vol. 3, Issue. 4, pp. 384-405, November 2009.

[5] R. Zemmari, U. Nickel, and W. Wirth, "GSM Passive Radar for Medium Range Surveillance," in European Radar Conf. EuRAD, pp. 49-52, September/October 2009.

[6] M. Villano, F. Colone, and P. Lombardo, "Antenna Array for Passive Radar: Configuration Design and Adaptive Approaches to Disturbance Cancellation," International Journal of Antennas and Propagation, 2013.

[7] F. Belfiori, S. Monni, W. van Rossum, and P. Hoogeboom, "Antenna Array Characterisation and Signal Processing for an FM Radio-Based Passive Coherent Location Radar System," IET Radar, Sonar \& Navigation, vol. 6, no. 8, pp. 687-696, 2012.

[8] M. Alam, K. Jamil, Z. Alhekail, and S. Al-Humaidi, "A multi-band multi-beam software-defined passive radar Part II: Signal processing," in IET Conference Publications, 2012.

[9] D. K. P. Tan, M. Lesturgie, H. Sun, and Y. Lu, "Spacetime interference analysis and suppression for airborne passive radar using transmissions of opportunity," IET Radar, Sonar \& Navigation, vol. 8, Iss. 2, pp. 142152, February 2014

[10] D. Poullin, "Passive detection using digital broadcasters (DAB, DVB) with COFDM modulation," IEE Proc.-Radar Sonar Navig., vol. 152, no. 3, June 2005.

[11] F. Colone, "A Multistage Processing Algorithm for Disturbance Removal and Target Detection in Passive Bistatic Radar," IEEE Transaction on Aerospace and Electronic Systems, vol. 45, no. 2, April 2008.
[12] R. Tao, H. Wu, and T. Shan, "Direct-path suppression by spatial filtering in digital television terrestrial broadcasting-based passive radar," IET Radar, Sonar and Navigation, vol. 4, Issue. 6, pp. 791-805, November 2009.

[13] G. Chabriel, J. Barrère, G. Gassier, and F. Briolle, "Passive Covert Radars using CP-OFDM signals: a new efficient method to extract targets echos," in RADAR 2014, IEEE International Conference, (Lille), pp. 1-6, October 2014.

[14] F. Pipon, F. Delaveau, and D. Heurguier, "Une approche "communication" pour la détection de cibles mobiles," Revue de l'Electricité et de l'Electronique, vol. 1, pp. 26-35, January 2006.

[15] S. Searle, J. Palmer, L. Davis, D. O'Hagan, and M. Ummenhofer, "Evaluation of the ambiguity function for păssive radar with OFDM transmissions," in 2014 IEEE Radar Conference, (Cincinnati, OH), pp. 1040-1045, 19-23 May 2014.

[16] J. Palmer, H. Harms, S. Searle, and L. Davis, "DVB-T Passive Radar Signal Processing," IEEE Transactions on Signal Processing, vol. 61, no. 8, pp. 2116-2126, April 2013.

[17] H. Harms, L. Davis, and J. Palmer, "Understanding the signal structure in DVB-T signals for passive radar detection," in IEEE Radar Conference, (Washington, DC), pp. 532-537, May 2010.

[18] G. Gassier, G. Chabriel, J. 'Barrère, F. Briolle, and C. Jauffret, "A Unifying Approach for Disturbance Cancellation and Target Detection in Passive Radar using OFDM," IEEE Transactions on Signal Processing, vol. 64, no. 22, pp. 5959-5971, November 2016.

[19] C. Berger, B. Demissie, J. Heckenbach, P. Willett, and Z. Shengli, "Signal Processing for Passive Radar Using OFDM Waveforms," IEEE Journal of Selected Topics in Signal Processing , vol. 4, no. 1, pp. 226238, Feb. 2010.

[20] ETSI Standard : EN 300744 V1.5.1 Digital Video Broadcasting (DVB). [Online] Available: http://www.etsi.org/deliver/etsi_en/300700_300799/ 300744/01.05.01_40/en_300744v010501o.pdf.

[21] G. Gassier, F. Briolle, J. Barrère, and G. Chabriel, "Pilot-Aided Beamforming for OFDM Detection Over Severe Multipath Fading," in 21st IEEE International Conference on Electronics Circuits and Systems, (Marseille), pp. 247-250, December 2014.

[22] D. Poullin, M. Flecheux, and M. Klein, "Elevation angle estimation for low-altitude targets using DVB (SFN broadcasters)," IEEE Aerospace and Electronic Systems Magazine, vol. 27, pp. 27-35, January 2012.

[23] D. Poullin and M. Flecheux, "Passive 3D tracking of low altitude targets using DVB (SFN Broadcasters))," IEEE Aerospace and Electronic Systems Magazine, vol. no. 27, pp. 36-41, January 2012.

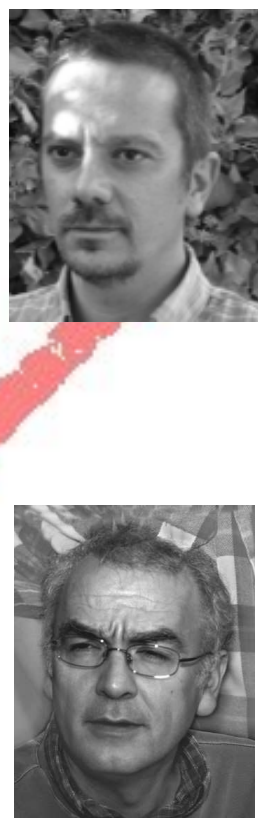

Gilles Chabriel received the Microelectronic and Automatic Engineer diploma from Polytech'Montpellier, France, in 1992 and the Ph.D. degree in Physics from University of Toulon (UTLN), France, in 1997. He is currently an Assistant Professor in the Sciences and Applied Sciences Department of UTLN, where he teaches Applied Mathematics, Electronics and Signal Processing. In the Signals and Systems Team of the IM2NP, his research is in the areas of signal processing, telecommunication, passive radar and electronic systems. of IM2NP Laboratory, Université de Toulon (UTLN), France, where his research interests include wave separation, array processing and passive radar.
Jean Barrère was born in Bayonne, France, in 1961. He received the Ph.D. degree in Mechanics from the University of Bordeaux, France, in January 1990. From 1990 to 1994, he worked for ELFAquitaine (currently Total-Fina-Elf), CERFACS (European Centre for Research and Advanced Training in Scientific Computation), and Aerospatiale (currently Airbus), all in Toulouse, France. His work focused on filtration laws in porous media studies and then plane structure design. $\mathrm{He}$ is currently a research engineer in the Signals and Systems Team 